\title{
EMPLOYEE SALARY PREDICTION USING MULTI MODEL MACHINE LEARNING TECHNIQUES: A COMPARATIVE ANALYSIS
}

\author{
KRISHNA SAI, JATIN \& SPOORTHI, SREESH
}

B.Tech. students of Dayananda Sagar University, Kudlu Gate, Bangalore, India

\begin{abstract}
Generally, job advertisements don't mention the salary for a particular designation. So, this project is used to predict the salary of an employee based on certain parameters like designation, skillsets \& his/her experience in the industry. The project is done by comparing each parameter of every individual so that the salary of a person can be predicted. The project is implemented using Python code \& Machine learning models. In the front end, a web page will be created to show the predicted salary, this is done by HTML, CSS. In the backend, the model is trained with multiple ML models and compared based on various parameters like accuracy and algorithm performance. Algorithms are Logistic Regression with the accuracy of $31 \%$, Decision Tree with 100\% \& Random Forest with $100 \%$. Based on the algorithm performance, a random forest is more efficient than a decision tree. So it is used for deployment. Later, for future developments, we can predict the salary of all the employees from a particular company at once.

KEYWORDS: Salary prediction, Machine Learning, Python, Pandas, Matplotlib, Hyper Parameter Tuning, Grid Search CV, Joblib
\end{abstract}

Received: Nov 10, 2020; Accepted: Dec 01, 2020; Published: Dec 26, 2020; Paper Id.: IJCSEITRDEC20209

\section{INTRODUCTION}

The purpose of this project is to predict an employee salary prediction using various ML algorithms. This project is mainly used by HR companies to determine the salary. Our professors supported us throughout this project. This project is useful for the employees and as well as employers. As this project predicts the salary of an employee based on their designation, skills, working experience, various factors, it can be used by anyone who wants to know their salary. It can also be used by any student who needs to know his/ her capability by predicting their salary for their future job, by giving their skills and other parameters as input.

In this project, the software/libraries we used are python and its libraries, machine learning algorithms, hyperparameter tuning using grid search $\mathrm{CV}$, and flask server.

The main purpose of predicting employee salary is to ease the employee's job in selecting their future job and to create a convenient application for employers. We will have a web server supporting many parameters of employees to predict their salary. Above all, we hope to provide a comfortable user experience along with accurate results. In this model there is a small risk involved while predicting an employee's salary. There is a small chance of predicting the wrong salary. But there is a very small percentage of chance for this error, as we have defined our model in such a way that it will avoid this type of error. 


\section{METHODOLOGY}

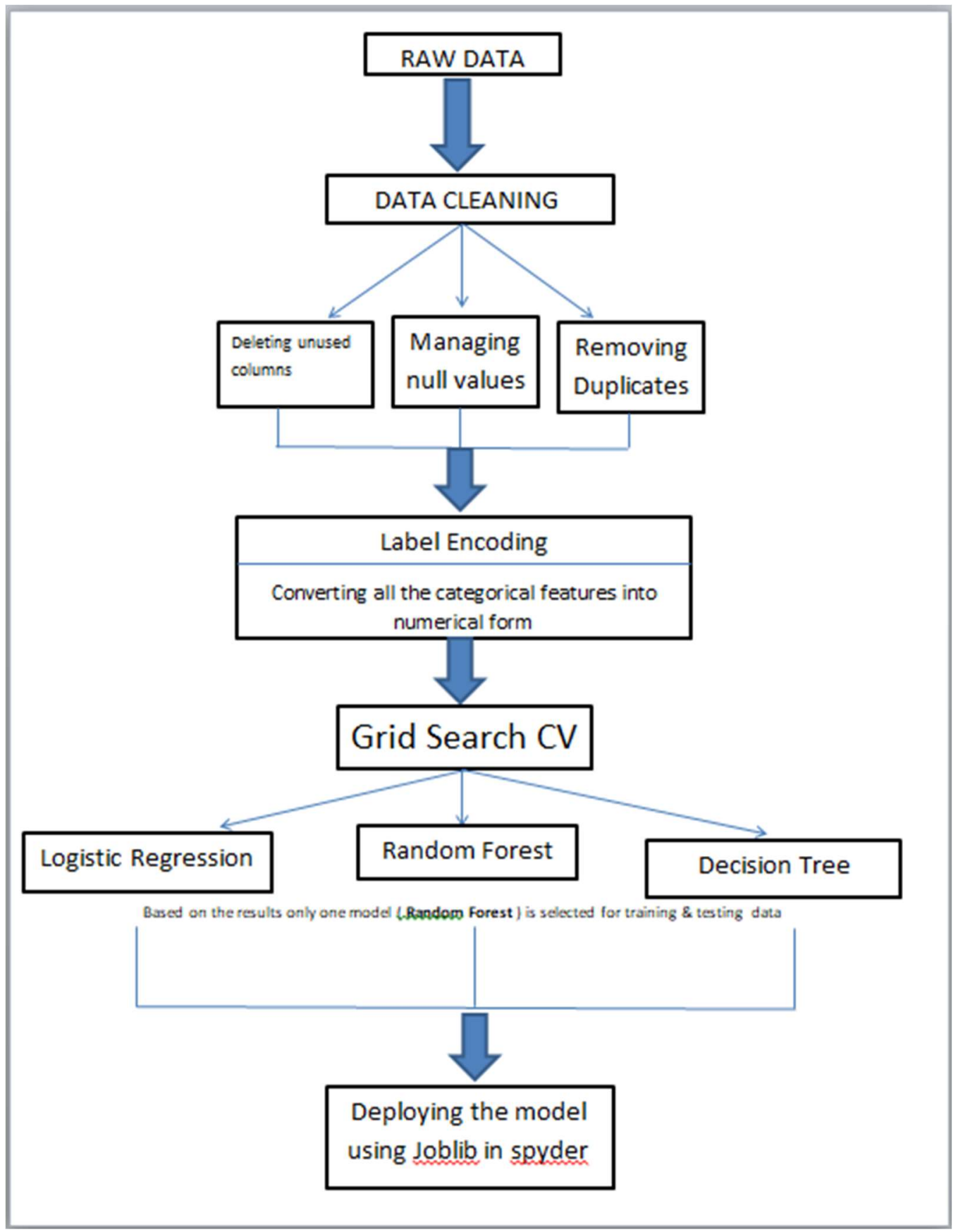

The dataset comprises around 14000+ rows with 6 columns. To gain useful insights we compare and contrast various strategies from the dataset. The following points will describe more about the dataset.

1. Data Collection: Data is collected from the Kaggle datasets. It consists of each categorical associated numerical option that is helpful to predict the remuneration of a worker.

2. Data Cleaning: Data has been cleaned by many pre-processing techniques during this section. Checking for any null values and filling them, redundant options and columns that don't seem to be used for the prediction of remuneration are removed. 
3. Feature Engineering: Feature choice algorithms are needed to convert categorical options into numerical types. Label cryptography is employed during this model to try to do the task.

4. Dataset Description: applied math tools and straightforward models are utilized to supply a preliminary and compact description of the information.

5. Feature Selection: Feature choice algorithms are used to mechanically choose the foremost informative options within the dataset regarding the output category.

6. Model Selection: A grid search CV is performed to search out the best hyper-parameters for a collection of well-known machine learning models. This can be not solely wont to notice the most effective model however additionally the most effective parameters with the best accuracy.

7. Model coaching \& Validation: the chosen models are trained and cross-validated to search out the classifiers that best describe the information and can predict the output variable with the best scores

The \{raw knowledge|data|information\} collected isn't helpful while not correcting the associate correct preprocessing section to get rid of the sources of noise and normalize the remaining data. One should additionally decide the way to treat the missing values. During this case, samples with missing values were substituted by an empty string, as there are solely eight null values in our dataset.

In our work, we tend to don't contemplate the keywords that seem but five times (corresponding to zero.10\% of the entire range of job posts). This ends up in the removal of many options that may be termed as noise. A very important feature is preprocessing which has additionally been given to the interpretation of categorical options into numerical options.

In our dataset at the start the expertise column is within the style of a string that's within the vary, so changing this string into the float is critical. The designation, department \& key skills are categorical options. To coach the information exploitation of any machine learning model, categorical options have to be compelled to be reborn into numerical options. Label cryptography is employed for this job. Encode categorical options as a one-hot numeric array.

The label encoder will be wont to normalize labels. It may be wont to rework non-numerical labels (as long as they're hashable and comparable) to numerical labels. Rework labels back to original cryptography. When changing the whole dataset within the numerical type, our dataset is prepared for coaching. Before coaching the model, hyperparameter standardization and grid search cv should be performed. When making a machine learning model, you will be conferred with style decisions on the way to outline your model design.

Oftentimes, we do not forthwith grasp what the best model design ought to be for a given model, and therefore we'd wish to be able to explore a variety of prospects. In true machine learning fashion, we'll ideally raise the machine to perform this exploration and choose the best model design mechanically. Parameters that outline the model design are remarked as hyperparameters and therefore this method of checking out the perfect model design is remarked as hyperparameter standardization. Grid search CV may be a methodology wont to train \& check the information in an associate distinctive manner.

By exploiting this methodology we will make sure that each part of the information is going to be trained and it makes it simple whereas testing. Grid search is the method of acting hyperparameter standardization to work out the best 
values for a given model. This can be vital because the performance of the whole model is predicated on the hyperparameter values given.

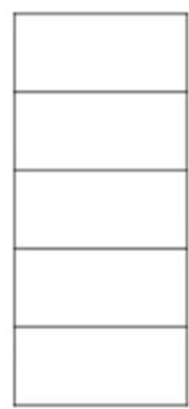

100 samples
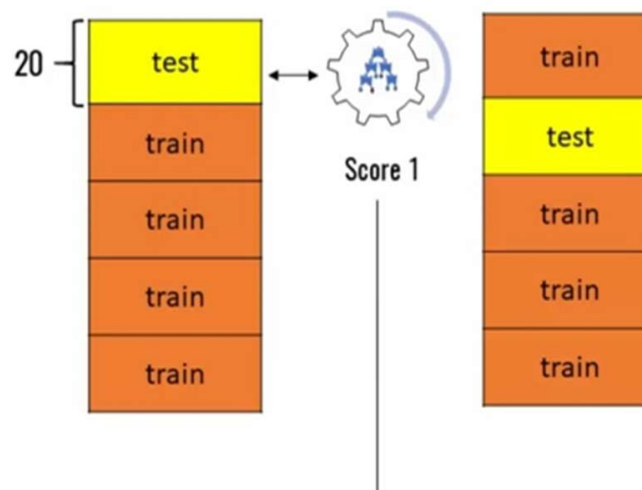

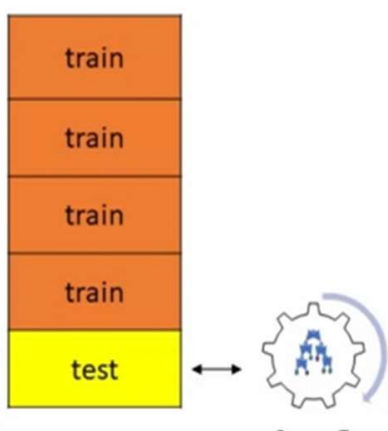

Score 5

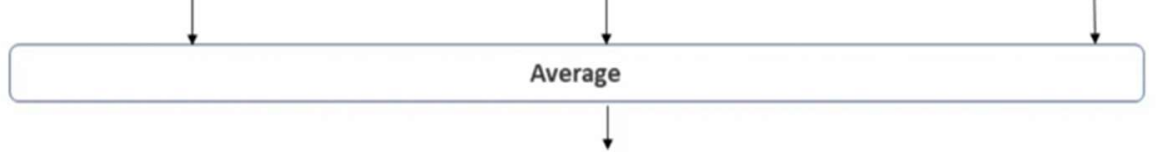

Average Score

Consider the above example, if there are one hundred samples within the dataset and $\mathrm{cv}=$ five in grid search $\mathrm{cv}$ then, it'll divide the whole dataset into five equal components. within the 1 st split, it'll check the first half and train remaining components, then within the second split, it'll check the second half and train remaining components and then on until the fifth split. At the tip, the typical score is taken into account from the five scores and it'll be terribly correct. By exploiting this grid search CV, we will even notice the most effective models and best parameters with the best accuracy. To search out the most effective model there will be several parameters that may be thought-about. The most issue we've to think about is accuracy, if we've two or models with similar and best accuracy then we tend to contemplate the performance of the formula and by the method, it works.

\section{DATASET INSPECTION}

Relation between each feature and the salary can be explained visually through scatter plot.
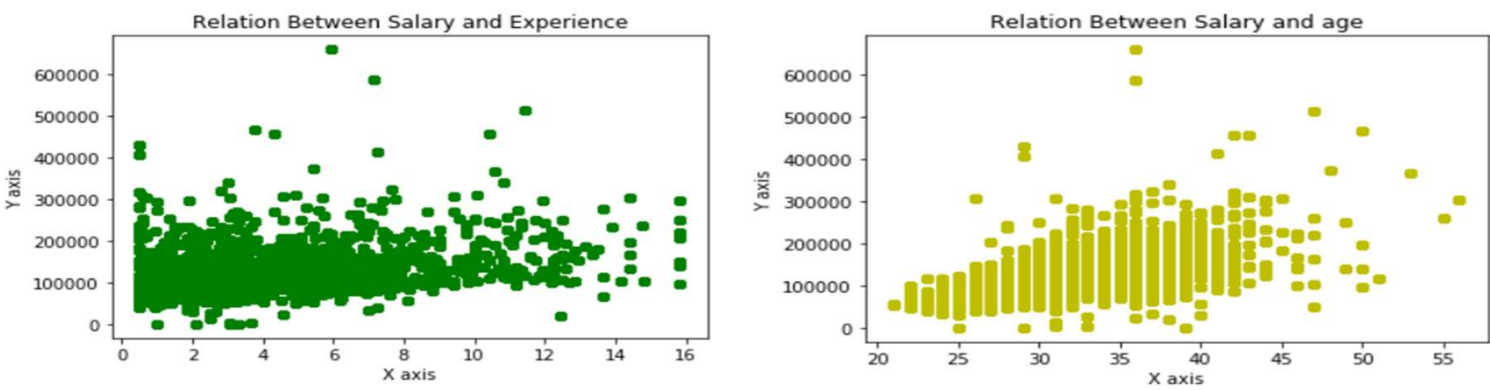

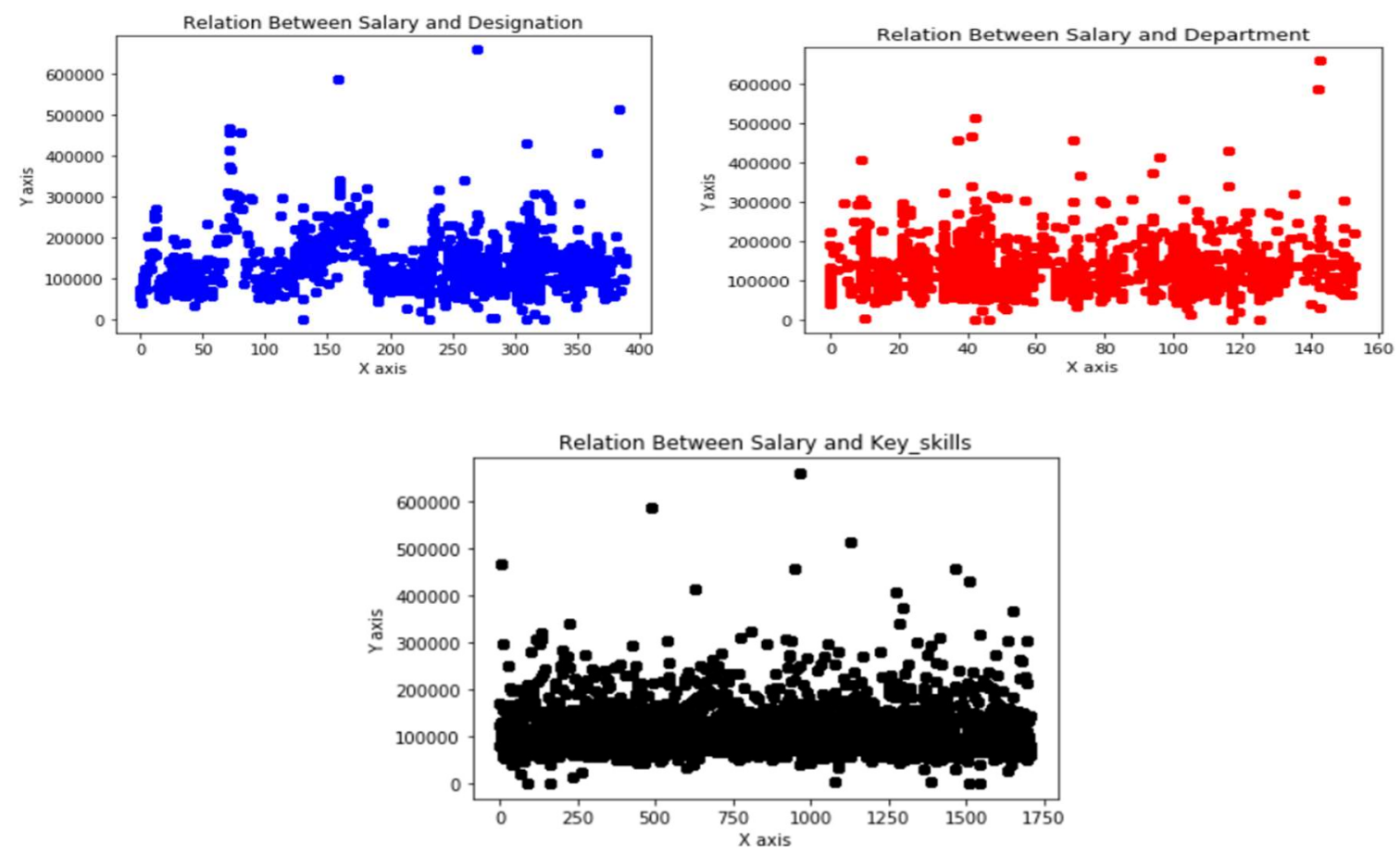

In Our Dataset, there are 5 important features that are useful to predict the salary. From the above scatter plots it is evident that all the features are almost equally important in order to predict the salary, so each and every feature should be considered while training the model. Though the above graphs did not give us clear information about how important the features are, we can still understand some parts of the dataset.

From the Experience \& Salary graph, we can tell that the salary is not completely proportional to the experience, as the highest salary person has around 6 years of experience whereas the highest experience in the dataset is around 16 years. It's applicable in the other way too; the person with the least salary is not always whose experience is the least. In Fact, the salary of many people with the least experience is more than the salary of many people with higher experience.

From the age \& Salary graph, we can tell that the salary is not completely proportional to the age, as the highest salary person is of 35 years of age, whereas the oldest person in the dataset is around 55 years. But many times age is proportional to age, which can be clearly observed from the above graph. Only a few people have higher salaries at a young age and lower salaries at old age.

From the other 3 graphs, we can only tell that designation, department and key skills are very important to predict the salary. As we have used label encoding to convert these categorical features into numerical form, we cannot understand much from these graphs. 


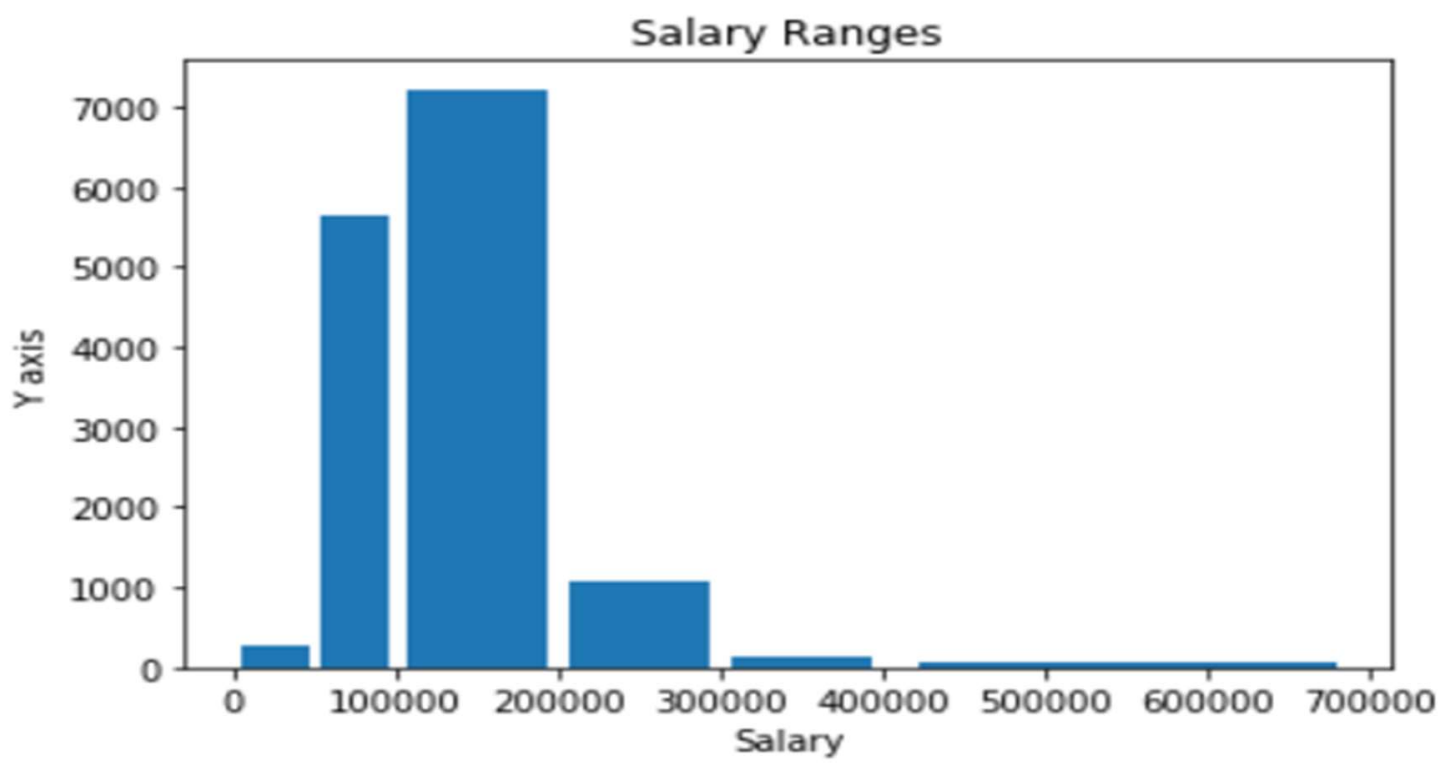

To understand more about the salary column which is the target column, we need to find the salary ranges and should find how many exist in a particular range. From the above histogram, we can observe that there are around 7000 people whose salary is between 1 lakh to 2 lakhs per month in our dataset, that just means half of the people from the dataset are having a good amount of salary based on their skills and other parameters. There are more than 5000 people who are getting salaries from 50,000 to 1 lakh and very few people with less than 50,000. Looking at the other people who are earning more than 2 lakh and under 3 lakh they are also around 1000 people which is a decent number of people with such huge salaries. Few people are earning between 3 lakhs and 4 lakhs and very few people are earning above 4 lakhs per month. These are the insights that we can draw from the above graph.

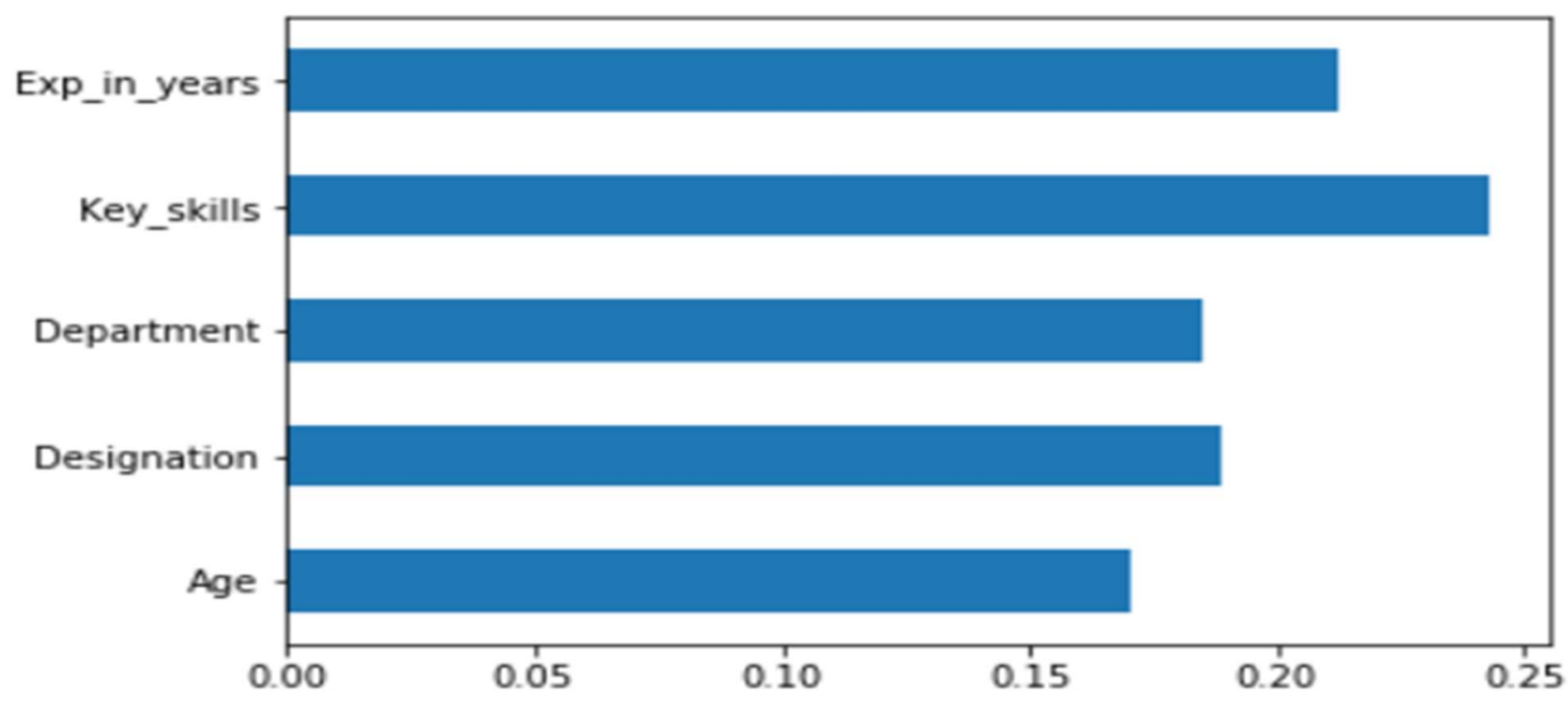

From the above bar graph, we can easily say that the column key skills are more important to predict the salary, but at the same time, even the other columns are also not too far. So even with this bar chart, we cannot tell how much exactly a column is important to predict the salary. The only thing we can understand is that the order of effectiveness of columns is key skills, experience, designation, department, age. 


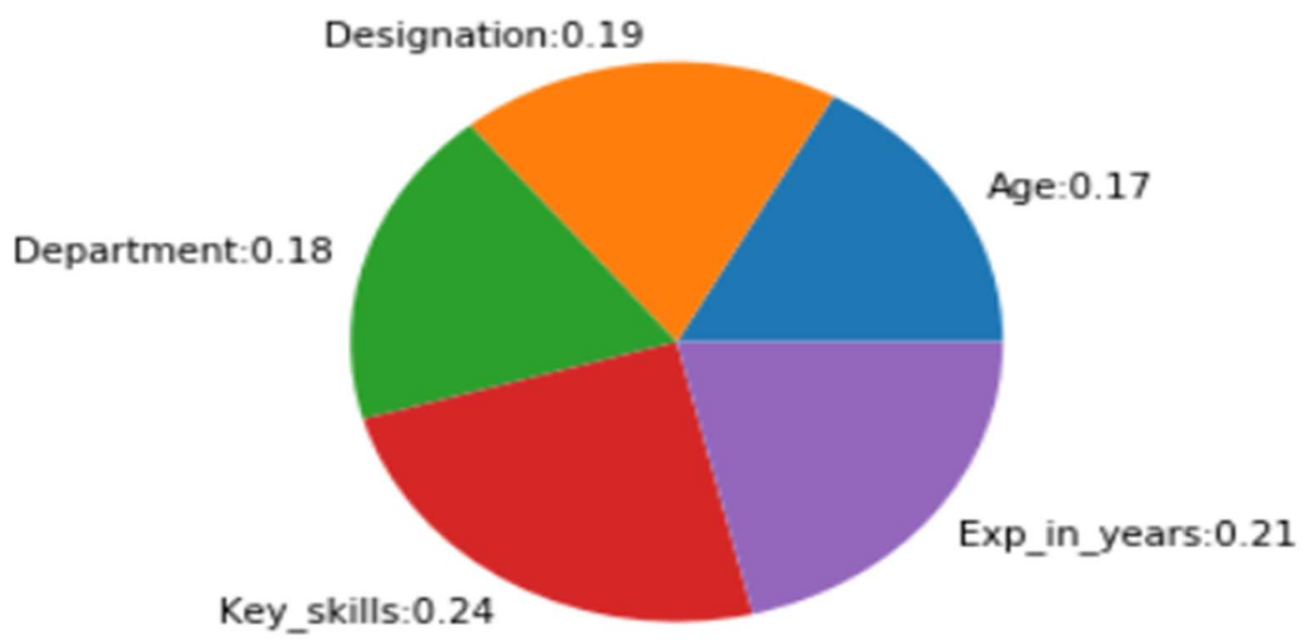

To understand the columns completely, the above Pie Chart is drawn.

As discussed Earlier, Key skills are most important among all the columns, from the above pie chart it can be said that it has $24 \%$ importance which is the highest among all and the age has the least importance with $17 \%$. This simply means that every parameter is very important, as there is only a small difference between the highest and the least percentages.

For suppose let's say if a parameter has above $50 \%$ and the combination of the remaining all parameters is around $50 \%$, in that case, we can observe that 1 or more parameters with very less percentages can even be omitted from the dataset if they are too low. But now our dataset is completely fine and all the parameters are important in predicting the salary.

Usually, a heatmap is drawn to find these important features, but from the pie chart, it's clear that all are important. So we can just see how heatmap will help us in telling the important features if we can't understand it with bar graphs or pie charts. 


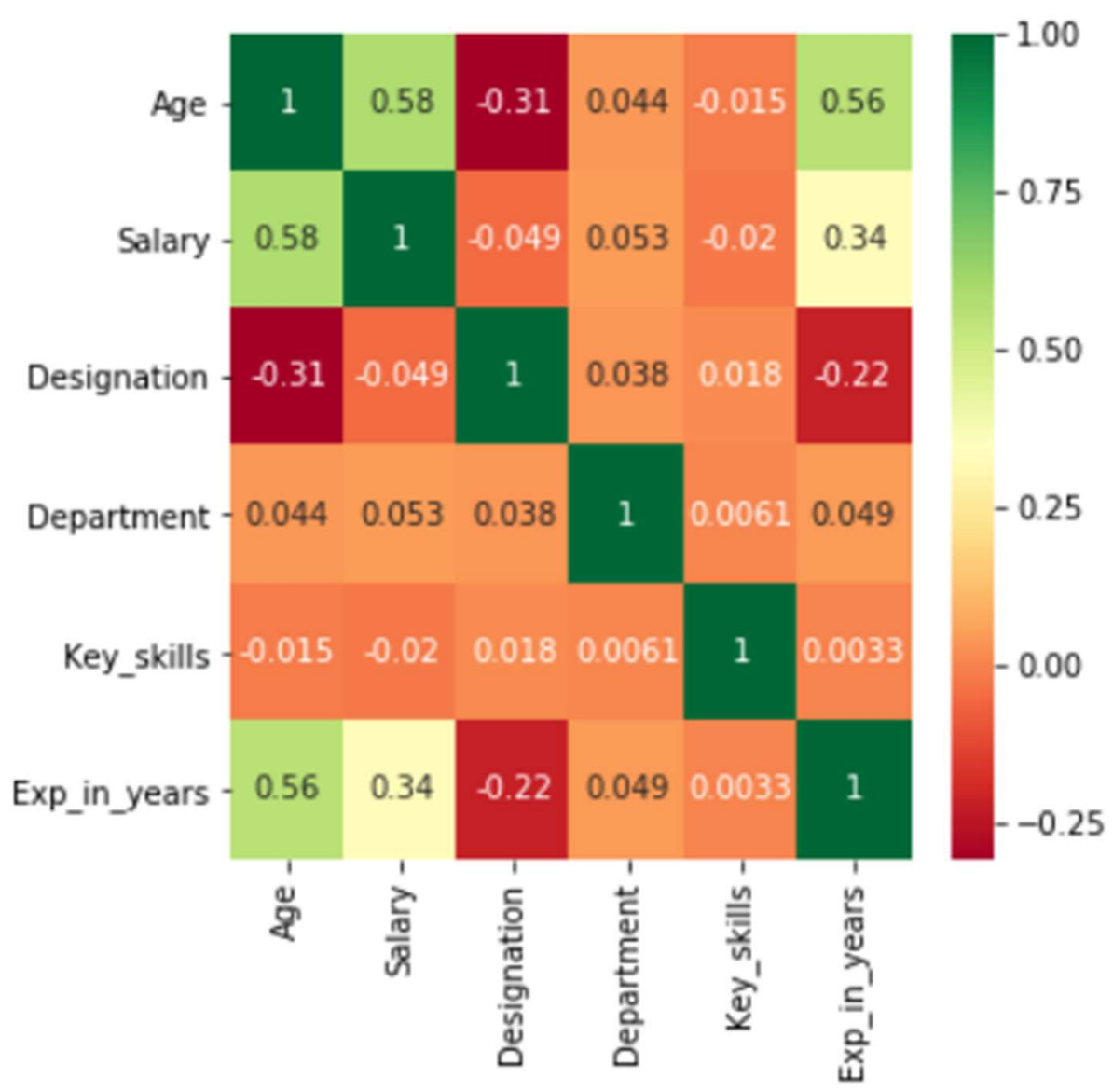

The above heatmap is just drawn for reference, there's no use of a heat map for our problem. But if any row or column of a heatmap has all the values less than 0.01 except the one which is matching itself, then we can omit that particular row or column. Here every row and column has a value more than 0.01 , so every parameter is considered for model training.

\subsection{Logistic Regression}

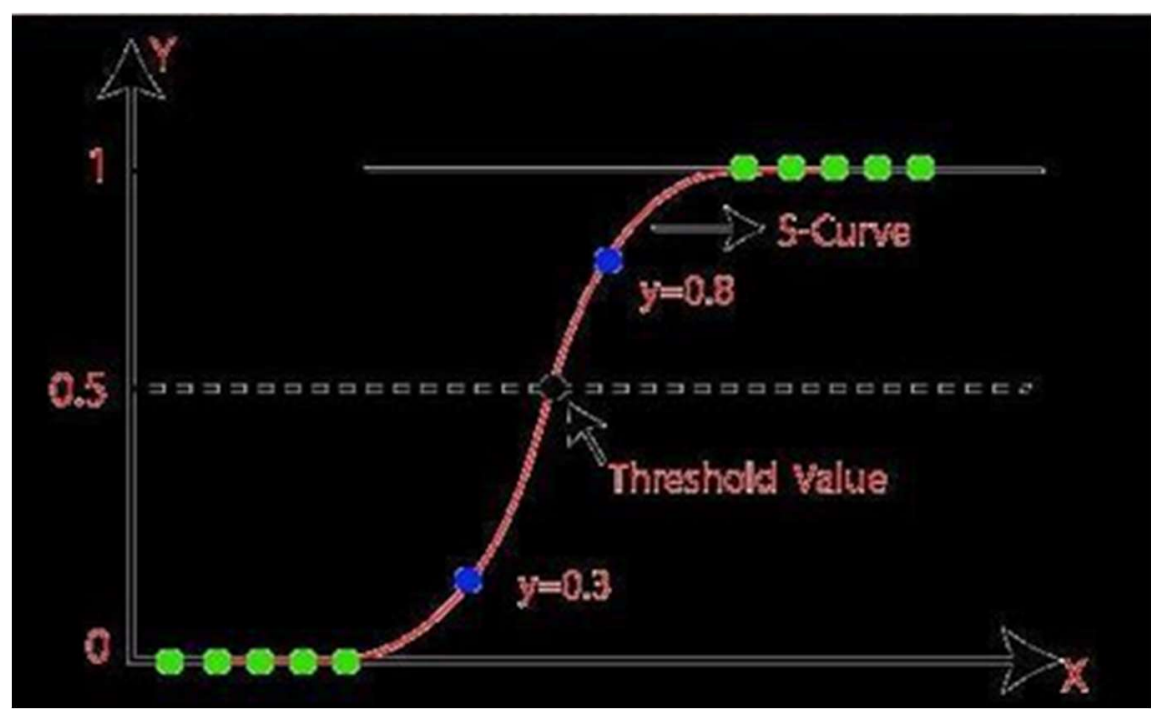

Figure 3.1: Representation of Logistic Regression 
Logistic Regression is a model which in its basic form uses a logistic function to model a binary dependent variable, even though there are various compound adjuncts. During the regression analysis, logistic regression (also called logit regression) is meant to evaluate the variables of logistic models (one of the forms of binary regression). Logistic Regression is comparatively effortless to administer, elucidate and very efficient to train. This model makes no presumptions about distributions of classes in featured space. It is rapid at categorising undefined records.

Logistic Regression assesses the association between the specific variable and one or more independent variables by approximating prospects with the utilization of a logistic function; it's the cumulative distribution function of logistic distribution. Hence it handles the similar set of complications as forbid regression using alike methods, which later leads to use of cumulative normal distribution curve. Identically, in the dormant variable elucidations of these two processes, the first presumes a standard logistic distribution of fallacy and the second a standard normal distribution of fallacy.

Logistic Regression can be considered a distinctive case of mainstream linear models and hence cognate to linear regression. The representation of logistic regression, although is made on various presumptions of linear regression. To be precise the cue distinctions among these two models could be viewed through these two attributes of logistic regression.

\subsection{Decision Tree}

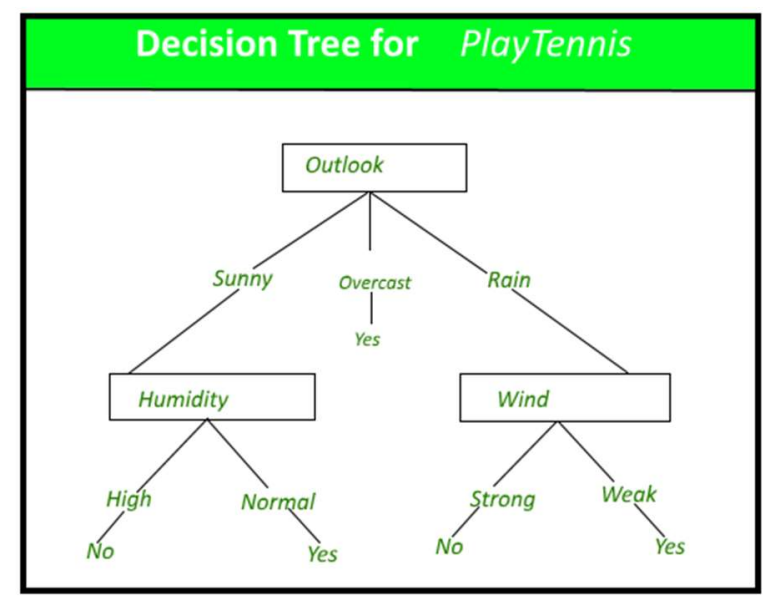

A Decision tree can be called as a decision support tool. It uses a decision model similar to a tree structure, which shows possible consequences and it also includes chance event outcomes, resource costs, utility. It is one of the ways to display an algorithm that uses conditional control statements. Decision trees are most likely to be used in research operations to be more specific in decision analysis which would help to identify a strategy that could help the team to reach a goal.

\section{A decision tree will have three types of nodes:}

Decision Nodes are the type of nodes in a particular activity at which the tree branches into several other operations. It is represented using squares. Chance Nodes are the type of nodes that represents the probabilities of an occurrence of certain results. It is represented using circles. End Nodes, as the name, specifies, is the end unit or the peripheral unit of a tree. It is represented using triangles. 
A decision tree can be understood by dividing the source section into subdivisions based on attribute values. This process should be repeated on each obtained subdivision in a recursive form which is also termed as recursive partitioning. The recursive method is completed when the subdivisions at every node have a similar value of the target variable. Another condition when splitting further would not add any value to predictions. For constructing a decision tree, a classifier would not require any domain knowledge or parameter setting and hence is appropriate for probe knowledge discovery. The decision tree would be able to handle high dimensional data. To conclude decision tree classifiers possess good accuracy. The introduction of a decision tree is an ideal approach to obtain knowledge on classification.

\subsection{Random Forest}

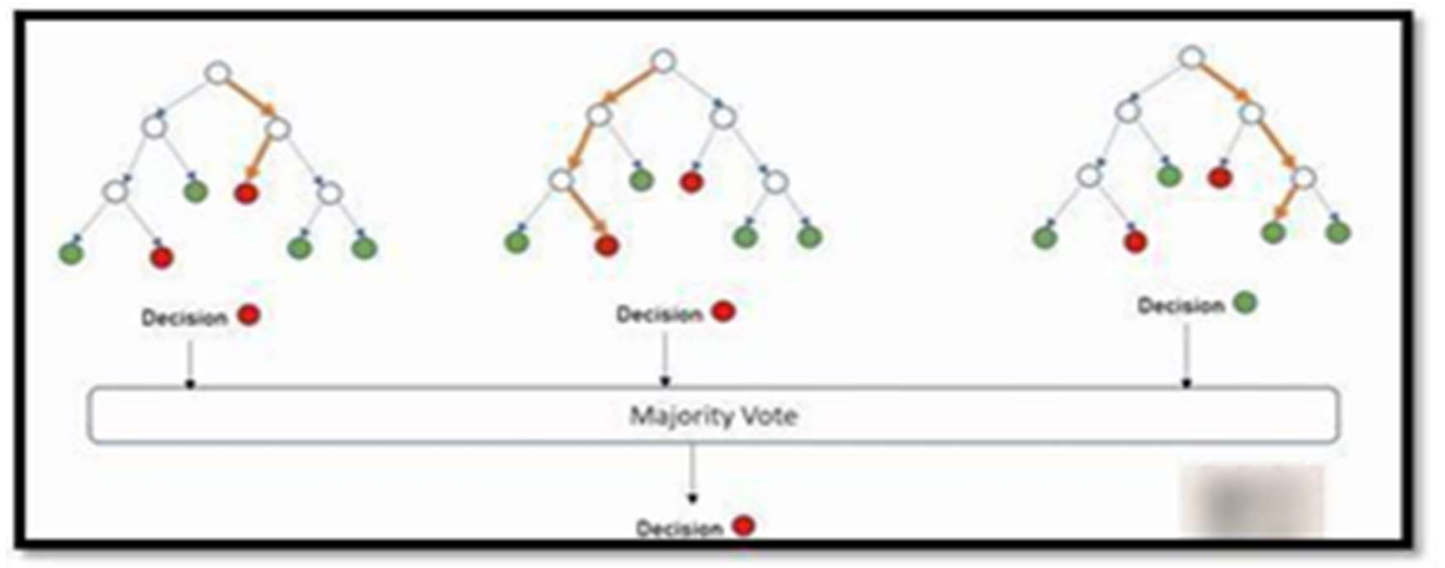

Figure 3.3: Representation of Random Forest

Decision trees are among the popular methods for numerous machine learning chores. Educating about decision trees is considered an important prerequisite to serving as an off the sill procedure for data mining.

Particularly trees that have grown very deep tend to exhibit highly irregular patterns, they are over fixed to training sets that have low bias but high variance. Random Forest is one of the ways to equate multiple deep decision trees. They should be trained in numerous parts of similar training sets, which clearly implies our goal is to reduce variance. This change could be expensive as it leads to a small increase in bias and also a slight loss of interpretability but in turn, it will boost the efficiency of the model.

Random forests are holding the efforts of decision tree algorithms together. It considers the teamwork of many decision trees hence ameliorating the performance of every single random tree. Forest projects the effect of K-fold crossvalidation but it cannot be considered as a similar effect.

Random Forest has a group of learning methods for classification, regression, and various other chores that operate by establishing a mess of decision trees at training time and presenting the output class as a mode of classes or mean/average probabilities of the individual trees. Random forests normally outperform decision trees, but their precision is lower than the gradient of the individual trees. Random Forests are recurrently used as "Blackbox" models in businesses since they generate rational predictions over a good range of knowledge by ensuring essential configuration in packages like sci-kit-learn

Random Forest Algorithm has an appreciable quality that it is effortless to measure the relative importance of every feature on the prediction. Sklearn supplies a significant tool to measure a feature's significance by considering how 
much the tree nodes utilize that feature to turn down adulteration across all trees in the forest. It calculates this score automatically for every feature after training and scales the outcome so the total of the whole importance should equate to one.

\section{MODEL COMPARISON}

\section{Logistic Regression}

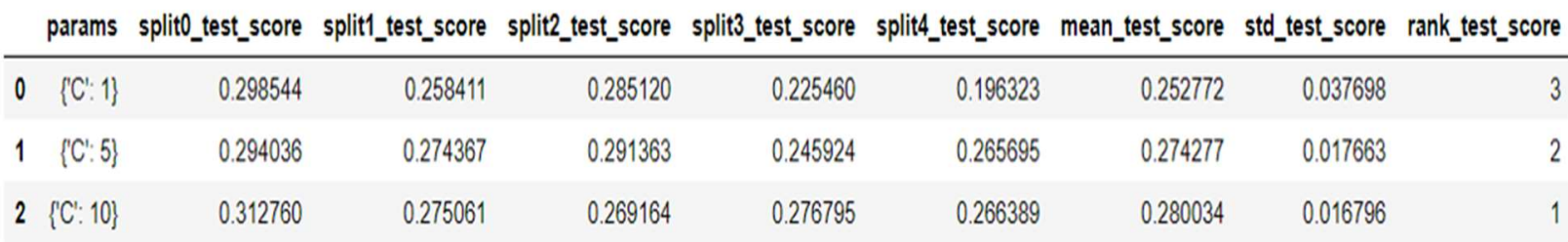

The above table shows the result of the model trained with logistic regression. Though the parameters are changing there's no rise in the accuracy of the model. The highest accuracy which can be observed from the above table is just $31 \%$ where $\mathrm{C}=10$ and the highest average score is just $28 \%$. As the accuracy is very less, this model cannot be used for deployment.

\section{Decision Tree}

\begin{tabular}{|c|c|c|c|c|c|c|c|c|c|}
\hline & params & split0_test_score & split1_test_score & split2_test_score & split3_test_score & split4_test_score & mean_test_score & std_test_score & rank_test_score \\
\hline 0 & $\begin{array}{l}\text { \{'criterion': 'gini', } \\
\text { 'splitter': 'best'\} }\end{array}$ & 1.0 & 1.0 & 1.0 & 1.0 & 1.0 & 1.0 & 0.0 & 1 \\
\hline 1 & $\begin{array}{r}\text { \{'criterion': 'gini', } \\
\text { 'splitter'; } \\
\text { 'random'\} }\end{array}$ & 1.0 & 1.0 & 1.0 & 1.0 & 1.0 & 1.0 & 0.0 & 1 \\
\hline 2 & $\begin{array}{r}\text { \{'criterion': } \\
\text { 'entropy', } \\
\text { 'splitter': 'best'\} }\end{array}$ & 1.0 & 1.0 & 1.0 & 1.0 & 1.0 & 1.0 & 0.0 & 1 \\
\hline 3 & $\begin{array}{l}\text { \{'criterion': } \\
\text { 'entropy', } \\
\text { 'splitter': } \\
\text { 'random'\} }\end{array}$ & 1.0 & 1.0 & 1.0 & 1.0 & 1.0 & 1.0 & 0.0 & 1 \\
\hline
\end{tabular}

The above table shows the result of the model which is trained with Decision Tree. The accuracy of this model is $100 \%$ irrespective of the parameters. Considering the accuracy this model is the best with $100 \%$ accuracy, but in the future if there is any need in adding more columns to the dataset then there can be change in accuracy as the algorithm is not very powerful.

\section{Random Forest}

params splito_test_score split1_test_score split2_test_score split3_test_score split4_test_score mean_test_score std_test_score rank_test_score

\begin{tabular}{|c|c|c|c|c|c|c|c|c|c|}
\hline 0 & $\begin{array}{r}\left\{n_{-} \text {estimators': }\right. \\
1\}\end{array}$ & 0.999307 & 0.998959 & 0.996185 & 0.997572 & 0.996878 & 0.99778 & 0.001194 & 3 \\
\hline 1 & $\left\{\begin{array}{r}\left\{n \_ \text {estimators': }\right. \\
5\}\end{array}\right.$ & 1.000000 & 1.000000 & 1.000000 & 1.000000 & 1.000000 & 1.00000 & 0.000000 & 1 \\
\hline 2 & $\begin{array}{r}\left\{n_{-} \text {estimators': }\right. \\
10\}\end{array}$ & 1.000000 & 1.000000 & 1.000000 & 1.000000 & 1.000000 & 1.00000 & 0.000000 & 1 \\
\hline
\end{tabular}

The above table shows the result of the model trained with Random Forest. The accuracy of the model is 100 when the value of $n \_$estimators is 5,10 . As the accuracy is maximum even this model is the best in terms of accuracy. If 
there is any need of adding more columns to the dataset then the accuracy can't be predicted, but a random forest algorithm is better than a decision tree as it is the combination of multiple decision trees. So this model can be deployed.

\section{MODEL DEPLOYMENT}

Machine learning is a well-known process in which it is mostly used for prediction. There are numerous algorithms available in different libraries that can be used for prediction. We built a prediction model on data based on surveys using different machine learning algorithms, results are taken after testing data on various Machine learning models of Prediction.

\section{Deployment using Flask:}

Flask is a Python-based tool used for developing small scale websites/webpages. Flask is very flexible to make API using python. As of now, we have developed a model 'omg. ml' which can predict the salary of an employee based on various attributes of the data.

A web application is made, in which the user needs to input all the values which will be fed to the model. Now based on the training of the model, it will predict the salary of that person / Employee. As the model fed with a random forest algorithm, it gives $100 \%$ accuracy, there is a very minute chance of the wrong prediction, if the inputs are given within the dataset then there is no chance of error at all.

\section{HTML Form -}

Predicting the salary from various attributes we need to collect the data (values of new attribute) and then use the Random Forest model/ algorithm which has already been fit to predict salary. So to collect the data we have created an HTML form that will contain the various options to select from each attribute. So, we have created an HTML form. 


\section{Flask Script -}

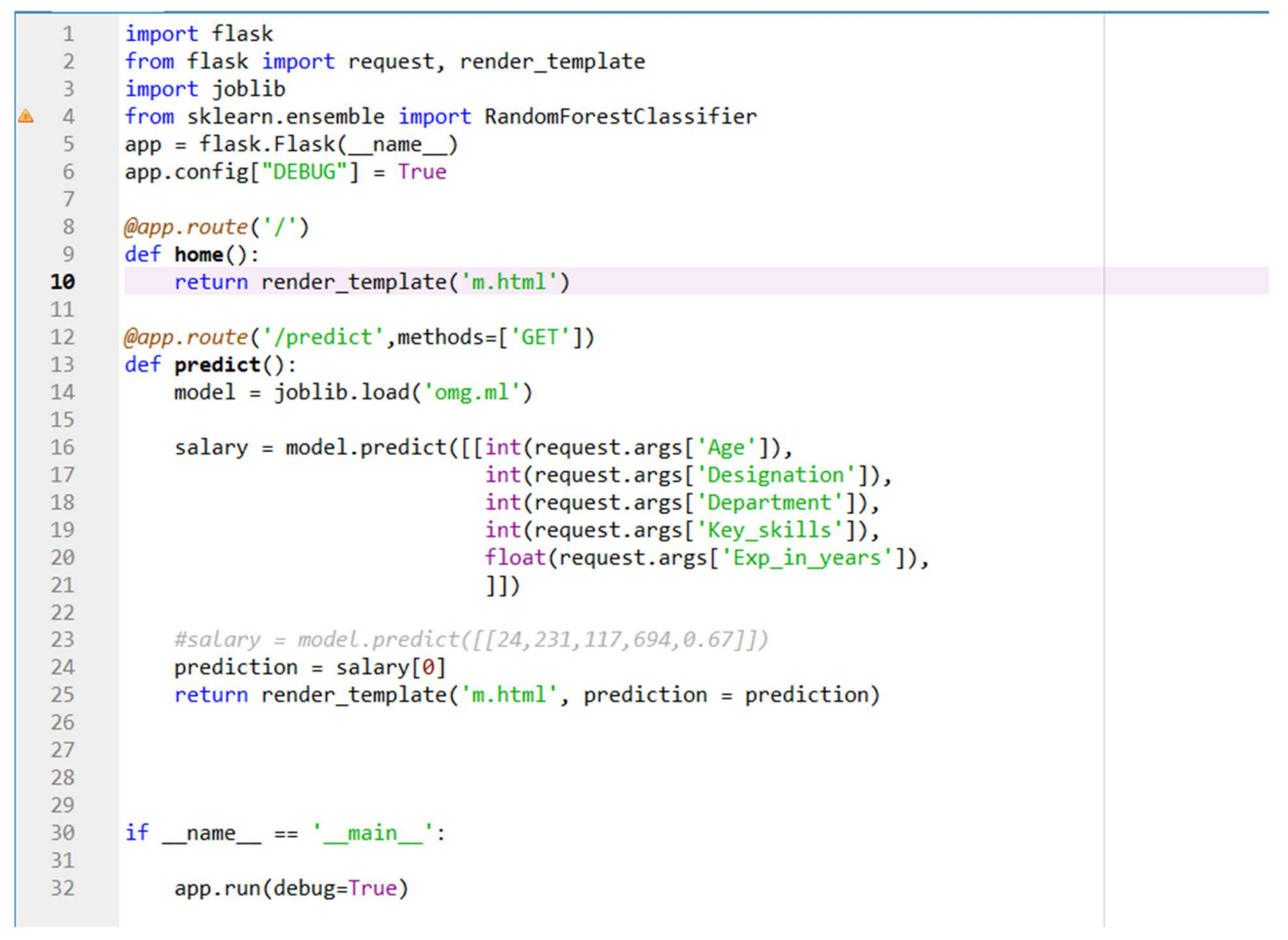

\section{Predicting Salary}

Once the form is submitted by a candidate the web page should display the predicted value of salary. For this, we require the model file (omg. ml) which is created in the same folder.

Here, after values are stored in a variable (i.e., salary) to be predicted in the form of a dictionary. Converted it into a list of the dictionary's values and passed it as an argument for the predict function. In this function, we load the omg.ml file and predict the new values and return the predicted salary. To run this on the localhost open the terminal of the required environment through anaconda navigator and type the following commands:

Python rf.py

Then the IP address of localhost appears on the screen, copy the link address and paste in the browser to launch the user interface. 
6:ar. C:\windows \system32\cmd.exe - python rf.py

$-\square \times$

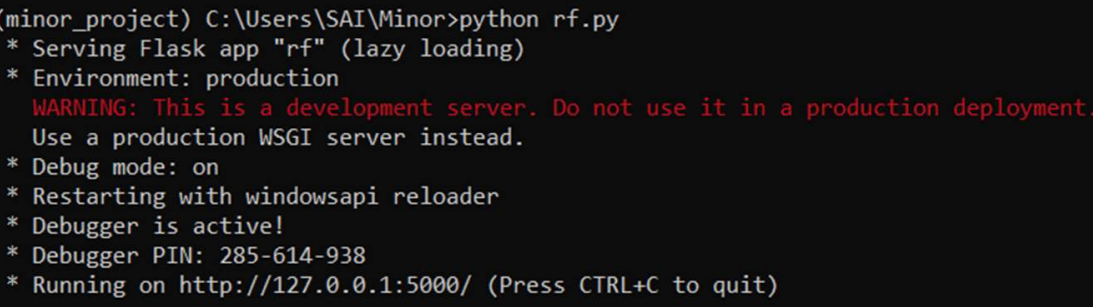

The user interface appears on the localhost and we need to fill in the details and click on predict to predict the salary.

\section{CONCLUSIONS}

As we have used 3 different techniques for our model such as decision tree, random forest classifier and got accuracies of 1.0, 1.0 and 0.5 respectively. As we got a decision tree and random forest as best accuracy among all hence random forest is taken to DEPLOY our model. For deployment we use flask server and SpyderRun the model on a local host.

Through the survey taken, we assess the features that can be used to predict salaries. Results indicate that some features, such as experience, skills or certain job roles contribute significantly to the final salary expected by employees. Finally, the model predicted salary with zero experience (Fresher) and experienced employee with a difference in their respective salaries. An employee with many skill sets and zero experience predicted a bit less salary when compared with an experienced employee with same skill sets. 


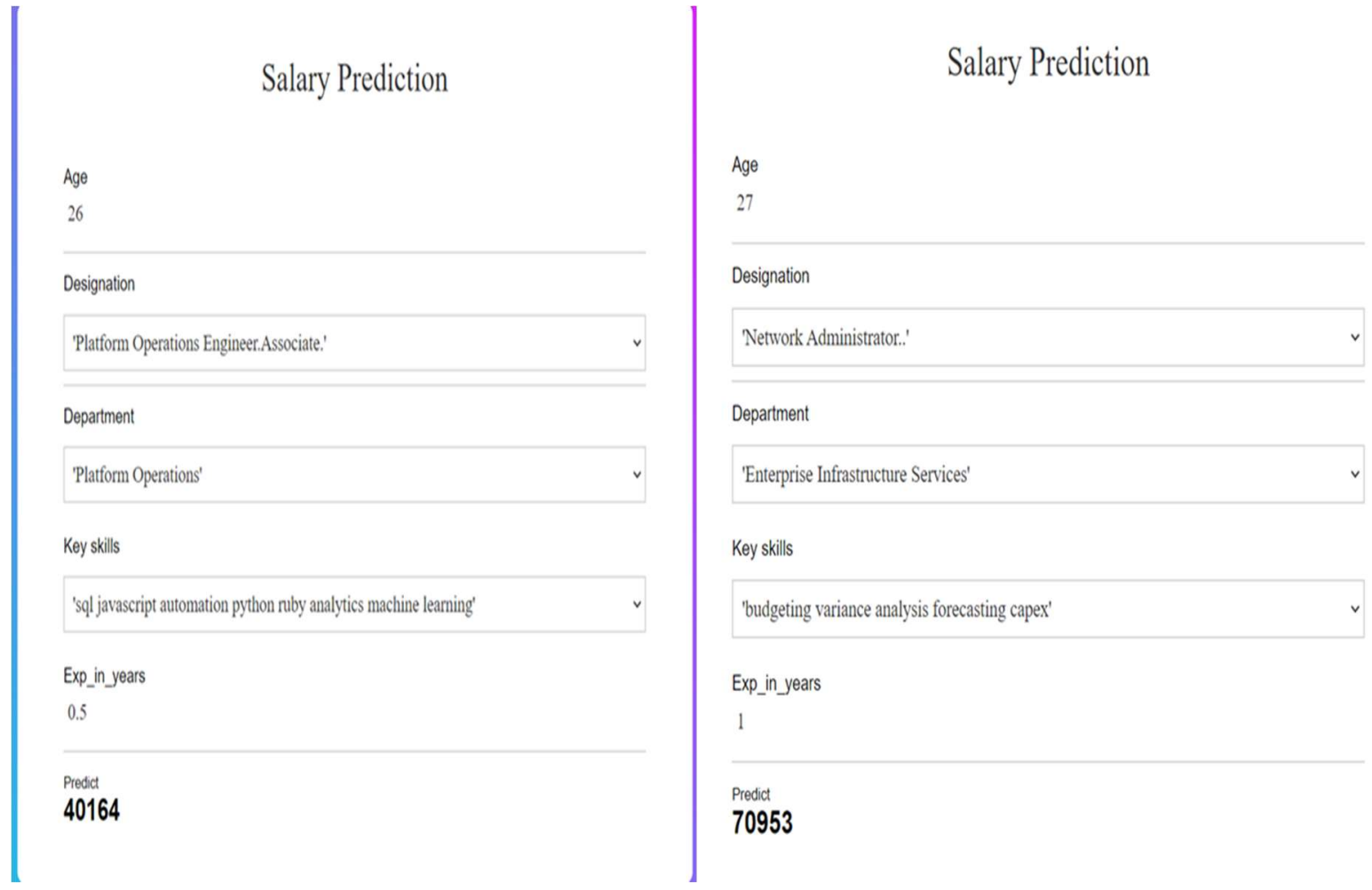

Salary Prediction

\begin{tabular}{l} 
Age \\
\hline $26 \mid$ \\
\hline Designation \\
\hline 'Support Delivery Manager..' \\
\hline Department \\
'AmaTec - SDM' \\
Key skills \\
'amazon e - commerce portal handling business analysis' \\
Exp_in_years \\
0.6 \\
\hline Predic \\
\hline 74542
\end{tabular}

\section{REFERENCES}

1. https://www.ijcseonline.org/spl_pub_paper/IJCSE-ICACICT-2019-16.pdf

2. https://www.scribd.com/document/398535833/Implement-of-salary-prediction-system-to-improve-student-motivation-usingdata-mining-technique-pdf 
3. https://www.cs.ubc.ca/ nando/540-2013/projects/p58.pdf

4. https://www.cvfoundation.org/openaccess/content_cvpr_2014/papers/Kazemi_One_Millisecond_Face_2014_CVPR_paper.pdf

5. $\quad$ https://www.jmlr.org/papers/volume13/biau12a/biau12a.pdf

6. https://www.researchgate.net/publication/259235118 Random Forests and Decision Trees

7. https://www.atlantis-press.com/journals/ijcis/25899235/view\#abstract-0

8. http://www.ijitee.org/wp-content/uploads/papers/v9i6/F4545049620.pdf https://www.researchgate.net/publication/320319964_Parameter_Tuning in_Random_Forest_Based_on_Grid_Search_Metho d for Gender Classification Based on Voice Frequency

9. https://stackabuse.com/cross-validation-and-grid-search-for-model-selection-in-python

10. https://www.mygreatlearning.com/blog/label-encoding-in-python

11. https://github.com/

12. https://www.javatpoint.com/

13. https://www.geeksforgeeks.org/machine-learning/

14. Danthala, S. W. E. T. H. A., et al. "Robotic Manipulator Control by using Machine Learning Algorithms: A Review." International Journal of Mechanical and Production Engineering Research and Development 8.5 (2018): 305-310.

15. Durgabai, R. P. L., and P. Bhargavi. "Pest Management using Machine Learning Algorithms: A Review." International Journal of Computer Science Engineering and Information Technology Research (IJCSEITR) 8.1 (2018): 13-22.

16. Kumar, Y. Jeevan Nagendra, and TV Rajini Kanth. "GIS-MAP Based Spatial Analysis of Rainfall Data of Andhra Pradesh and Telangana States Using R." International Journal of Electrical and Computer Engineering 7.1 (2017): 460.

17. Chandolikar, N. S., and V. D. Nandavadekar. "Investigation of Feature Selection and Ensemble Methods for Performance Improvement of Intrusion Attack Classification." International Journal Of Computer Science And Engineering (IJCSE) Vol 2: 131-136. 